Bull. Austral. Math. Soc.

VOL. 37 (1988) [423-428]

\title{
QUASI-CONTINUITX WITH RESPECT TO SEMI-REGULARISATION TOPOLOGY
}

\author{
STANISLAW PSYK
}

\begin{abstract}
This paper gives some sufficient conditions under which upper, lower or both upper and lower quasi-continuity of multifunction in the process of semi-regularisation of a topological space are preserved. Analogous results for continuous maps are true.
\end{abstract}

A subset $A$ of a topological space $X$ is said to be semi open if there exists an open set $U$ such that $U \subseteq A \subseteq \bar{U}[5]$. It is easy to see that $A$ is semi open if and only if $A \subseteq \overline{\operatorname{Int} A}$. A subset $B$ of a topological space $(X, \tau)$ is called regular open if $B=\operatorname{Int} \bar{B}[2]$. It is well known that the family of all regular open sets forms a base for a smaller topology $\tau_{s}$ on $X$, called the semi-regularisation of $\tau$. The topological space $(X, \tau)$ is called semi-regular if $\tau=\tau_{s}$.

In the class of all topologies which have the same families of semi open sets as the topology $\tau$, there exists the finest topology $\tau_{\alpha}[7$, Corollary 1]. All topologies from the above class determine the same families of regular open sets [7, Proposition 6], so they have the same semi-regularisations, but the topology $\tau_{\alpha}$ and its semi-regularisation can have different families of semi open sets (see [7] or the topology $\tau$ in Example 1).

The symbols $\bar{A}^{i}, \operatorname{Int}_{i} A$ are used to denote closure and interior of the set $A$ in the space $\left(X, \tau_{i}\right)$. The symbol " $i$ "',nay be replaced by " $\alpha$ " or "s" or may be omitted for topological spaces $\left(X, \tau_{\alpha}\right),\left(X, \tau_{s}\right)$ or $(X, \tau)$ respectively.

The topological spaces are not assumed to satisfy any separation axioms, and regularity does not include the $T_{1}$ axiom. All notions and symbols which are not defined in this paper are used as in Engelking [2].

Let $F$ be a multivalued map which to each point $x \in X$ assigns a non-empty subset $F(x)$ of a topological space $Y$ (for simplicity we will write $F: X \rightarrow Y$ ). For any set $A \subseteq X, B \subseteq Y$ we will write [1]

$$
\begin{aligned}
F(A) & =\cup\{F(x): x \in A\}, \\
F^{+}(B) & =\{x \in X: F(x) \subseteq B\}, \\
F^{-}(B) & =\{x \in X: F(x) \cap B \neq \emptyset\} .
\end{aligned}
$$

Received 13 August 1987

Copyright Clearance Centre, Inc. Serial-fee code: 0004-9729/88 \$A2.00+0.00. 
A multivalued map $F: X \rightarrow Y$ is said to be:

- upper (respectively lower) quasi-continuous at a point $x_{0} \in X$ if for each open set $V \subseteq$ $Y$ such that $F\left(x_{0}\right) \subseteq V$ (respectively $F\left(x_{0}\right) \cap V \neq \emptyset$ ) and for each open neighbourhood $U$ of $x_{0}$ there exists an open non-empty set $U_{1} \subseteq U$ such that $F(x) \subset V$ (respectively $F(x) \cap V \neq \emptyset)$ for each $x \in U_{1}[\boldsymbol{\theta}]$,

- upper (respectively lower) almost continuous at a point ${ }_{0} \in X$ if for each open set $V \subseteq Y$ such that $F\left(x_{0}\right) \subseteq V$ (respectively $F\left(x_{0}\right) \cap V \neq \emptyset$ ) we have $x_{0} \in$ Int $\overline{F^{+}(V)}$ (respectively $x_{0} \in \operatorname{Int} \overline{F^{-}(V)}$ ) $[10,11]$.

A multivalued map is said to be upper or lower quasi-continuous (almost continuous) if it has this property at each point.

In the sequel we will use the following equivalent conditions:

- a multivalued map $F: X \rightarrow Y$ is upper (respectively lower) quasi-continuous if and only if for any open set $V \subseteq Y$ the set $F^{+}(V)$ (respectively $F^{-}(V)$ ) is semi open,

- a multivalued map $F: X \rightarrow Y$ is upper (respectively lower) almost continuous if and only if for any open set $V \subseteq Y$ we have $F^{+}(V) \subseteq$ Int $\overline{F^{+}(V)}$ (respectively $\left.F^{-}(V) \subseteq \operatorname{lnt} \overline{F^{-}(V)}\right)[\mathbf{1 0}]$.

Any single-valued map $f: X \rightarrow Y$ can be considered as a multivalued map with values $\{f(x)\}$. In this case the above definitions give the definitions of quasi-continuity in the sense of Kempisty [4] (sometimes called semi-continuity [5]) and almost continuity in the sense of Husain [3].

The symbols: $Q_{u}(F, \tau), Q_{l}(F, \tau), A_{u}(F, \tau), A_{l}(F, \tau)$ are used to denote the sets of all points at which a multivalued map $F:(X, \tau) \rightarrow Y$ is upper or lower quasicontinuous or almost continuous respectively.

REMARKS 1: For topological spaces $(x, \tau)$ and $\left(X, \tau_{s}\right)$ we have:

(a) Int, $U \subseteq$ Int $U$ for every set $U \subseteq X$;

(b) $\bar{U} \subseteq \bar{U}^{s}$ for every set $U \subseteq X$;

(c) Int $B=$ Int $_{s} B$ for every $\tau$-closed set $B \subseteq X$;

(d) $\bar{A}^{s}=\bar{A}$ for every $r$-open set $A \subset X$;

(e) any $r_{s}$-semi open set is $\tau$-semi open;

(f) $\tau_{s}$-quasi-continuity implies $\tau$-quasi-continuity;

(g) Int $\bar{V} \subseteq$ Int $_{s} \bar{V}^{s}$ for every set $V \subseteq X$;

(h) $\tau$-almost continuity implies $\tau_{\mathrm{a}}$-almost continuity.

Proofs: The properties (a) and (b) follow from $\tau_{s} \subseteq \tau$. Now we are going to prove (c). Int $B$ is regular open, hence $\operatorname{Int} B=\operatorname{Int}_{s}(\operatorname{Int} B) \subseteq \operatorname{Int}_{s} B$. The converse inclusion Int $B \subseteq \operatorname{Int} B$ follows from (a). Property (d) follows from (c) applied to the set $X \backslash A$. If $V$ is $\tau_{s}$-semi open then from (d) and (a) we have $V \subset \overline{\operatorname{Int}_{s} V^{s}}=$ $\overline{\text { Int }_{s} V} \subset \overline{\text { Int } V}$. Thus (e) is shown. Moveover (e) implies (f). From (c) and (b) we have 
Int $\bar{V}=$ Int, $\bar{V} \subseteq \overline{\text { Int. } V}^{s}$ which proves (g). Finally the property (h) is an immediate consequence of $(\mathrm{g})$.

ThEOREM 1. Let $(X, \tau)$ be a topological space and $Y$ a regular one. If $F: X \rightarrow Y$ is a multivalued map with compact values, then $\operatorname{Int}_{s}\left[Q_{u}(F, \tau) \cap Q_{l}(F, \tau)\right] \subset Q_{u}\left(F, \tau_{s}\right)$.

Proof: Assume that $x_{0} \in \operatorname{Int}_{s}\left[Q_{u}(F, \tau) \cap Q_{l}(F, \tau)\right] \backslash Q_{u}\left(F, \tau_{s}\right)$. Then there exist an open set $W_{0} \supseteq F\left(x_{0}\right)$ and a $\tau_{s}$-open set $U_{0}$ such that $x_{0} \in U_{0} \subseteq \operatorname{Int}_{s}\left[Q_{u}(F, \tau) \cap\right.$ $\left.Q_{l}(F, \tau)\right]$ and the following condition holds:

(1) every non-empty $\tau_{s}$-open set $U \subseteq U_{0}$ contains a point $x_{u}$ such that $F\left(x_{u}\right) \not \subset W_{0}$.

Because $F\left(x_{0}\right)$ is compact and $Y$ is a regular space, there exists an open set $W \subset Y$ satisfying $F\left(x_{0}\right) \subseteq W \subseteq \bar{W} \subseteq W_{0}$. The point $x_{0} \in Q_{u}(F, \tau)$; hence for sets $U_{0}$ and $W$, there exists a non-empty, $\tau$-open set $M \subseteq U_{0}$ such that:

(2) $F(x) \subseteq W$ for every point $x \in M$.

The set $M$ can be represented in the form $M=U \backslash N$ [8, Theorem 4.5] where $U \subseteq U_{0}$ is $\tau$-regular open and $N$ is a $\tau$-nowhere dense set. In view of (1) there exists a point $x_{u} \in U$ such that $F\left(x_{u}\right) \cap(Y \backslash \bar{W}) \neq \emptyset$. Because $x_{u} \in Q_{l}(F, \tau)$ for the sets $Y \backslash \bar{W}$ and $U$ there exists a nonempty, $\tau$-open set $A \subseteq U$ such that:

(3) $F(x) \cap(Y \backslash \bar{W}) \neq \emptyset$ for every point $x \in A$.

The sets $A$ and $U$ are non-empty, $\tau$-open and $A \subseteq U$. So we have $A \cap(U \backslash N)=A \cap M \neq \emptyset$. This means that $A \cap M$ contains a point satisfying (2) and (3). This is the contradiction which finishes the proof.

Theorem 2. Let $(X, \tau)$ be a topological space and $Y$ a regular one. If $F: X \rightarrow Y$ is a multivalued map then $\operatorname{Int}_{s}\left[Q_{u}(F, \tau) \cap Q_{l}(F, \tau)\right] \subseteq Q_{l}\left(F, \tau_{s}\right)$.

Proof: Assume that $x_{0} \in \operatorname{Int}_{s}\left[Q_{u}(F, \tau) \cap Q_{l}(F, \tau)\right] \backslash Q_{l}\left(F, \tau_{s}\right)$. Then there exist an open set $W_{0} \subseteq Y$ such that $W_{0} \cap F\left(x_{0}\right) \neq \emptyset$ and a $\tau_{0}$-open set $U_{0}$ such that $x_{0} \in U_{0} \subseteq \operatorname{Int}_{s}\left[Q_{u}(F, \tau) \cap Q_{\imath}(F, \tau)\right]$ and:

(1) every non-empty $\tau_{0}$-open set $U \subseteq U_{0}$ contains a point $x_{u}$ such that $F\left(x_{u}\right) \cap W_{0}=\emptyset$.

Let us take any point $y_{0} \in F\left(x_{0}\right) \cap W_{0}$ and an open set $W$ for which $y_{0} \in W \subseteq \bar{W} \subseteq W_{0}$ holds. Because $x_{0} \in Q_{l}(F, \tau)$ there exists a $\tau$-open non-empty set $M \subseteq U_{0}$ satisfying:

(2) $\quad F(x) \cap W \neq \emptyset$ for every point $x \in M$.

Let $M I=U \backslash N$, where $U \subseteq U_{0}$ is a $\tau$-regular open set and $N$ is $\tau$ nowhere dense $\left[8\right.$, Theorem 4.5]. From (1) we have a point $x_{u} \in U$ such 
that $F\left(x_{u}\right) \subset Y \backslash \bar{W}$. The point $x_{u} \in Q_{u}(F, \tau)$, so there exists a $\tau$-open set $A \subseteq U$ such that:

(3) $F(x) \subseteq Y \backslash \bar{W}$ for every point $x \in A$.

The sets $A$ and $U$ are non-empty $\tau$-open and $A \subseteq U$. So we have $A \cap(U \backslash N)=A \cap M \neq \emptyset$, this means that $A \cap M$ contains a point satisfying (2) and (3) and this is the contradiction completing the proof.

From Theorems 1, 2 and Remarks 1(f) we immediately obtain:

Corollary 1. Let $(X, \tau)$ be a topological space and $Y$ a regular one. $A$ multivalued map $F: X \rightarrow Y$ with compact values is lower and upper quasi-continuous if and only if it is simultaneously lower and upper $\tau_{s}$-quasi-continuous.

Corollary 2. Let $\tau_{1}$ and $\tau_{2}$ be topologies on $X$ which have the same semiregularisations. If $Y$ is a regular space, then a single valued map $f: X \rightarrow Y$ is $\tau_{1}$-quasi-continuous if and only if it is $\tau_{2}$-quasi-continuous.

Observe that regularity of the space $Y$ in Theorems 1 and 2 cannot be replaced by semi-regularity.

EXAMPLE 1.. Let us take on the real line $\mathbf{R}$ the following topologies:

$$
\begin{aligned}
\tau & =\{U \subseteq \mathbf{R}: 0 \in U\} \cup\{\emptyset\} \\
\tau_{2} & =\{\emptyset,\{0\},\{2\},\{0,2\}, \mathbf{R}\}
\end{aligned}
$$

It is easy to see that topology $\tau_{2}$ is semi-regular and the semi-regularisation $\tau_{s}$ of $\tau$ is the indiscrete topology. The multifunction $F:(\mathbf{R}, \tau) \rightarrow\left(\mathbf{R}, \tau_{2}\right)$ given by the formula $F(x)=\{0\}$ for $x=2$ and $F(x)=\{x\}$ for $x \neq 2$ is upper and lower $\tau$-quasi-continuous but it is neither upper nor lower $\tau_{s}$-quasi-continuous.

If $F$ is upper or lower quasi-continuous only, then Corollary 1 may be false, as the following shows:

EXAMPLE 2. Let $N$ be the natural topology on $\mathbf{R}$ and let $\tau$ be the topology $\tau$ from Example 1. The multifunction $F_{1}:(\mathbf{R}, \tau) \rightarrow(\mathbf{R}, N)$ given by the formula $F_{1}(x)=\{0\}$ for $x \in(-\infty, 0)$ and $F_{1}(x)=<0,1>$ for $x \in(0,+\infty)$ has compact values and it is easy to verify that $F_{1}$ is upper $\tau$-quasi-continuous but it is not upper $\tau_{\text {- }}$-quasicontinuous. However the formula $F_{2}(x)=\{0\}$ for $x \in(-\infty, 0)$ and $F_{2}(x)=<0,1>$ for $x \in(0,+\infty)$ gives a multifunction which is lower $\tau$-quasi-continuous but is not lower $\tau_{s}$-quasi-continuous.

Stronger than Corollary 1 is the following: 
Theorem 3. Let $(X, \tau)$ be a topological space and $Y$ a regular one. Let, for $i=1,2,3, \tau_{i}$ be a topology on $X$ such that $\tau_{s} \subseteq \tau_{i} \subseteq \tau_{\alpha}$ (in general $\left(\tau_{i}\right)_{\alpha} \neq\left(\tau_{j}\right)_{\alpha} \neq$ $(\tau)_{\alpha}=\tau_{\alpha}$ and $\tau_{i} \not \subset \tau_{j}$ for $\left.i, j=1,2,3\right)$. A multivalued map $F: X \rightarrow Y$ with compact values is lower $\tau_{1}$-quasi-continuous and upper $\tau_{2}$-quasi-continuous if and only if it is simultaneously upper and lower $\tau_{3}$-quasi-continuous.

Proof: Because the semi-regularisation of $\tau_{\alpha}$ is the topology $\tau_{s}$ ([7] Proposition 6), hence from the inclusion $\tau_{s} \subseteq \tau_{i} \subseteq \tau_{a}$ and from Remark 1 (d) we have $\overline{\operatorname{Int}}_{i} A^{i} \subset$ $\overline{\operatorname{Int}} \boldsymbol{\alpha}^{s}={\overline{\operatorname{Int}_{\alpha} A}}^{\alpha}$ for any set $A \subseteq X$. This means that any $\tau_{i}$-semiopen set is $\tau_{\alpha^{-}}$ semiopen, so $F$ is simultaneously lower and upper $\tau_{\alpha}$-quasi-continuous and in virtute of Corollary 1 it is $\tau_{s}$-quasi-continuous. To this end we shall prove that the semiregularisation of $\tau_{i}$ is the topology $\tau_{s}$ and then apply Remark $1(f)$. If the set $B$ is $\tau_{i}$-regular open, then from $\tau_{s} \subseteq \tau_{i} \subseteq \tau_{\alpha}$ and from Remark $1(\mathrm{c})$ we have: Int $\alpha \bar{B}^{\alpha}=$ Int, $\bar{B}^{\alpha} \subseteq \operatorname{Int}_{i} \bar{B}^{i}=B=\operatorname{Int}_{\alpha} B \subseteq \operatorname{Int}_{\alpha} \bar{B}^{\alpha}$. This means $B=\operatorname{Int}_{\alpha} \bar{B}^{\alpha}$ is $\tau_{\alpha}$ (hence

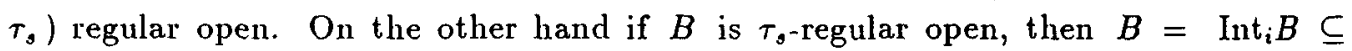
$\operatorname{Int}_{i} \bar{B}^{i} \subset \operatorname{Int}_{\alpha} \bar{B}^{s}=\operatorname{Int}_{s} \bar{B}^{s}=B$, so $B=\operatorname{Int}_{i} \bar{B}^{i}$ and the proof is complete.

Let us observe that if $\left(\tau_{1}\right)_{s}=\left(\tau_{2}\right)_{s}=\left(\tau_{3}\right)_{s}$ only, then Theorem 3 may be false as Example 2 shows together with the topology $\tau_{3}=\{U \subset \mathbf{R}: 3 \in U\} \cup\{\emptyset\}$. The multifunction $F_{1}$ is upper $\tau$-quasi-continuous and lower $\tau_{3}$-quasi-continuous, but it is neither lower nor upper $\tau_{3}$-quasi-continuous.

Now we will formulate some sufficient conditions under which upper (respectively lower) $\tau$-quasi-continuity implies upper (respectively lower) $\tau_{s}$-quasi-continuity.

TheOREM 4. Let $(X, \tau)$ be a topological space and $Y$ a regular one. If $F: X \rightarrow Y$ is a multivalued map with compact values, then $\operatorname{Int}_{s}\left[Q_{u}(F, \tau) \cap A_{l}(F, \tau)\right] \subset Q_{u}\left(F, \tau_{s}\right)$.

Proof: Just as in Theorem 4 we can prove that there exist sets $W, M=U \backslash N$ (where $W \subset Y$ is open $M \subseteq X$ is open, $U \subseteq X$ is regular open and $N \subseteq X$ is a nowhere dense set) and the point $x_{u} \in U$ for which $F\left(x_{u}\right) \cap(Y \backslash \bar{W}) \neq \emptyset$ and: (1) $F(x) \subseteq W$ for every point $x \in M$.

Because $x_{u} \in A_{l}(F, \tau), F^{-}(Y \backslash \bar{W})$ is $\tau$-dense in the set Int $\overline{F^{-}(Y \backslash \bar{W})}$ and consequently the set $G=U \cap F^{-}(Y \backslash \bar{W})$ is non-empty and dense in the set $H=$ $U \cap \operatorname{Int} \overline{F-(Y \backslash \bar{W})}$. Because $G \cap(H \backslash N) \neq \emptyset$ and $H \cap M=H \cap(U \backslash N)=$ $(H \cap U) \backslash N=H \backslash N$ we have $G \cap M \supseteq G \cap H \cap M \neq \emptyset$. But $F(x) \not \subset \bar{W}$ for every point $x \in G$, hence we have a contradiction to (1), so the proof is completed.

Using arguments similar to those in the proofs of Theorem 2 and 4 we can prove:

Theorem 5. Let $(x, \tau)$ be a topological space and $Y$ a regular one. If $F: X \rightarrow Y$ is a multivalued map then $\operatorname{Int}_{s}\left[A_{u}(F, \tau) \cap Q_{l}(F, \tau)\right] \subseteq Q_{l}\left(F, \tau_{s}\right)$. 
COROllary 3. Let $(X, \tau)$ be a topological space and $Y$ a regular one. If a multivalued map $F: X \rightarrow Y$ with compact values is upper quasi-continuous and lower almost continuous, then it is upper $\tau_{s}$-quasi-continuous.

Corollary 4. Let $(X, \tau)$ be a topological space and $Y$ a regular one. If a multivalued map $F: X \rightarrow Y$ is lower quasi-continuous and upper almost continuous, then it is lower $r_{b}$-quasi-continuous.

Observe that in Theorems 4 and 5 the assumption of lower or upper almost continuity cannot be weakened by lower or upper $\tau_{\theta}$-almost continuity, respectively. In Example 2 both multifunctions $F_{1}, F_{2}$ are upper and lower $\tau_{s}$-almost continuous but are neither upper nor lower $\tau_{s}$-quasi-continuous. Moreover Example 1 shows that the assumption of reqularity of the space $Y$ is essential.

REMARK 2: Theorems 1, 3, 4 will be true if we consider multifunctions with closed values in a normal space. This same applies to Corollaries 1 and 3 .

REMARK 3: If in all our considerations, beginning from Theorem 1, the term "quasi-continuity" is replaced by "continuity", similar results for continuous maps are obtained. In this case (for example) from Corollary 2 we have a theorem of Katetov [6, Lemma 2].

\section{REFERENCES}

[1] C. Berge, Espaces topologiques. Functions multivoques (Paris, 1966).

[2] R. Engelking, General topology (Warszawa, 1977).

[3] T. Husain, 'Almost continuous mappings', Prace Mat. 10 (1966), 1-7.

[4] S. Kempisty, 'Sur les functions quasicontinues', Fund. Math. 10 (1932), 184-197.

[5] N. Levine, 'Semi-open sets and semi-continuity in topological spaces', Amer. Math. Monthly 70 (1963), 36-41.

[6] M. Mrševič, I.L. Reilly and M.K. Vamanamurthy, 'On semi-regularization topologies', J. Austral. Math. Soc. Ser. A 38 (1985), 40-54.

[7] O. Njåstad, 'On some classes of nearly open sets', Pacific J. Math. 15 (1965), 961-970.

[8] J.C. Oxtoby, Measure and category (Springer-Verlag, New York Heidelberg Berlin, 1971).

[8] V. Popa, 'Asupra unei descompuneri a cvasicontinuitati multifunctiilor', Stud. Cerc. Mat. 27 (1975), 325-328.

[10] V. Popa, 'Asupra unor proprietātii ale multifunctiilor cvasicontinue si aproape continue', Stud. Cerc. Mat. 30 (1978), 441-446.

[11] R.E. Smithson, 'Almost and weak continuity for multifunctions', Bull. Calcutta Math. Soc, 70 (1978), 383-390.

Department of Mathematics

Pedagogical University

Arciszewskiego 22

76-200 Slupsk

Poland 